\title{
Revista de la
}

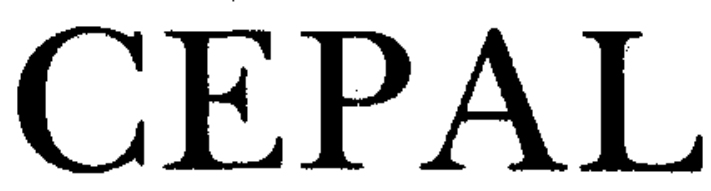

\author{
Secretario Ejecutivo \\ Norberto González \\ Secretario Ejecutivo Adjunto de \\ Desarrollo Económico y Social \\ Gert Rosenthal \\ Secretario Ejecutivo Adjunto de \\ Cooperación y Servicios de Apoyo \\ Robert T. Brown
}

\author{
Director de la Revista \\ Raúl Prebisch \\ Secretario Técnico \\ Adolfo Gurrieri \\ Secretaria Adjunta \\ Rosa Nielsen
}

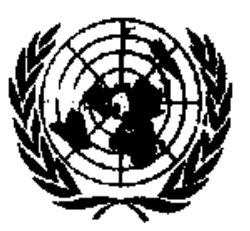

NACIONES UNIDAS

COMISION ECONOMICA PARA AMERICA LATINA Y EL CARIBE

SANTIAGO DE CHILE, AGOSTO DE 1985 


\section{Revista de la \\ C E P A L}

Santiago de Chile

Número 26

\section{SUMARIO}

Nota de la Dirección

Crisis y desarrollo en América Latina y el Caribe. Secretaria Ejecutiva de la ciPAL.

Exposición presentada a la Reunión de Expertos sobre Crisis y Desarrollo de América Latina y el Caribe. Enrique V. Iglesias

La periferia latinoamericana en la crisis global del capitalismo. Raúl Prebisch

Las perspectivas de la evolución política y social de América Latina. Torcuato Di Tella

La transformación del modelo de industrialización en América Latina.

Klaus Esser

El proceso de acumulación y la debilidad de los actores. Víctor E. Tokman

La crísis internacional y el desarrollo latinoamericano. Objetivos e instrumentos.

François Le Guay

La recuperación de la hegemonía norteamericana. María da Conceiçäo Tavares

Crisis, ajuste y politica económica en América Latina. David Ibarra

Comentario

Carlos Massad: "El costo real de la deuda externa para el acreedor y para el deudor" Revista de la CEPAL No 19, abril de 1983, pp. 185 à 197.

Observaciones acerca del análisis formal del servicio real de la deuda (Roger Lindquist y Soren Wibe)

Respuesia (Carlos Massad)

Publicaciones recientes de la CEPAL. 


\section{REVISTA DE LA CEPAL $\mathbf{N}^{\circ} 26$}

La crisis

internacional

y el desarrollo

latinoamericano. Objetivos

e instrumentos

\author{
François Le Guay*
}

Después de esbozar algunos de los efectos más notorios de la crisis actual, al autor presenta dos vias hipoteticas para salir de ella: la capitalista liberal y la socialdemócrata. Si bien la segunda vía le parece más favorable para el desarrollo futuro de los paises latinoamericanos, sostiene que incluso ella sería insufictente si no va acompañada del establecimiento en éstos de un patrón de desarrollo autónomo y autosustentado.

Durante los años setenta, tanto en la región como fuera de ella, se elaboraron muchas ideas teóricas y prácticas acerca de ese nuevo patrón de desarrollo, las que fueron oscurecidas por el reflorecimiento de la doctrina económica liberal. A juicio del autor, aquellas ideas mantienen su vigencia de modo que el problema actual no consiste en crear un nuevo paradigma sino en dar a la noción de desarrollo autónomo y autosustentado un carácter operacional, en especial en algunas áreas claves como la autosuficiencia en alimentos, el establecimiento de un proceso crecientemente autónomo de industrialización y la articulación de los secto. res industrial y agricola.

La operacionalización de las ideas orientadas a transformar el estijo predominante de desarrollo debe abarcar también a la planificación, la que deberja ser capaz de brindar una contribución más efectiva a la orientación del proceso en su conjunto. Entre los aspectos de la planificación que deberian mejorarse destaca la articulación de los horizontes temporales, el ensamble de la planificación global con la sectorial, y el desarrollo y consolidación de los procesos de concertación social.

Por último, afirma que en el campo de la integración tanto subregional como regiona! tampoco faltaron ideas, investigaciones $y$ hasta instituciones adecuadas; las causas de sus defectos y fracasos se deben atribuir, más bien, a la ausencia de voluntad politica para realizarlas, como lo mostrarian los escasos avances logrados en la integración industrial y energética.

*Profesor de ta Universidad de Gremble y del Instítulio de Altos Estudios de América Latina, de la Universidad de l"atis.

\section{Algunas reflexiones sobre los efectos de la crisis}

La manifestación más espectacular de la crisis -el nivel extraordinario de endeudamiento de la mayoría de los paises- ha centrado la atención internacional durante muchos meses. Después de la reunión del Fondo Monetario Internacional realizada en septiembre de 1984 , se produjo un relativo silencio en torno al asunto. Según fuentes autorizadas, la búsqueda de soluciones ad hoc para cada caso (el caso de México es el primer ejemplo) ha logrado suavizar el problema y el sistema financiero internacional ha superado el mayor peligro sin necesidad de cambios estructurales importantes. Esta me parece una visión exageradamente optimista. Aun en el aspecto meramente financiero me parece muy difícil afirmar que la situación actual, caracterizada por enormes flujos financieros netos de los países del Sur a los países del Norte, se pueda sostener por muchos años más.

Más importante que los aspectos financieros me parecen los efectos reales de la crisis en los sistemas económicos y en las estructuras sociales y políticas de los países en desarrollo. A nivel global, América Latina ha sufrido tres años de grave recesión (1981-1983), y el crecimiento de $2.5 \%$ del producto bruto en 1984 ni siquiera alcanza al crecimiento demográfico. El nivel de vida promedio ha retrocedido de 10 a 20 años, según los países.

Más grave aún, la crisis ha afectado en profundidad los sistemas productivos. En algunos países y en ciertos sectores de la agricultura o la industria, se ha traducido en desmantelamiento de capacidades productivas, atraso tecnológico y dispersión de mano de obra calificada. Dicho deterioro haría muy dificil en algunos sectores un crecimiento rápido aun si las condiciones de la demanda mejoraran.

En el aspecto social los impactos son también muy profundos y dejarán secuelas por períodos largos. Como consecuencia de la precariedad de los sistemas de protección social, la cesantía significa no solo una baja del poder adquisitivo sino una caída brutal en la miseria. El hambre en las ciudades y en el campo, y el deterioro de los 
servicios públicos de salud, vivienda y otros, tienen efectos irreversibles en la salud de las poblaciones. En resumen, la crisis ha acentuado los fenómenos de dependencia, desarticulación económica y social, y desigualdad entre regiones y grupos sociales, que son características conocidas del subdesarrollo. En otras palabras, ha exacerbado los elementos perversos de los estilos de desarrollo vigentes.

A ese respecto, la crisis ha hecho poco caso de las categorías en que estuvo de moda hace poco clasificar a los países del Tercer Mundo: exportadores de petróleo, nuevos países industrializados, paises de menor desarrollo relativo. Todos padecen deterioros estructurales graves. Si hay diferencias entre ellos, no es según las categorías mencionadas, sino según su grado de inserción en la economía mundial. En América Latina, entre los países más afectados figuran Argentina y Chile donde se practicaron políticas ultraliberales de apertura indiscriminada. En ambos se observan fenómenos de verdadera desindustrialización, con destrucción de sectores enteros, a veces establecidos hace muchos años.

Esos efectos ya se han analizado extensamente. En cambio, se ha examinado mucho menos un punto, a mi modo de ver, muy importante: los efectos de la crisis en el discurso político internacional y la evolución de las ideas. Es impresionante la poca capacidad de expresión internacional de los países en desarrollo, si se la compara con la del periodo de 1973 a 1980 . La voz colectiva del Tercer Mundo no se escucha más, como si los gobiernos hubieran quedado paralizados por el temor de perjudicar la solución posible de su situación individual frente a los países acreedores y los organismos financieros internacionales. Sin embargo, habría mucho que decir tanto sobre las responsabilidades que implica la situacion presente como sobre la validez permanente de las soluciones propiciadas por la comunidad internacional en los años setenta. La crisis no ha mostrado la obsolescencia de estas últimas sino, al contrario, la necesidad más urgente de ponerlas en marcha.

Pero no sólo el discurso político está paralizado sino también la evolución de las ideas en el campo económico, social y político. Aun los círculos académicos e intelectuales parecen inhibidos frente a la arrogancia de la "nueva ortodoxia" neoclásica. Es paradójico que los principales responsables de la situación logren imponer a sus víctimas, además de las consecuencias prácticas de la crisis, la aceptación de su propia visión de los acontecimientos.

A partir de 1984 ese panorama bastante sombrío comenzó a manifestar algunas señas más positivas. A nivel político, la más importante fue la realización de una serie de reuniones iniciadas en el mes de enero por la Conferencia Económica Latinoamericana convocada en Quito por el entonces Presidente del Ecuador, Doctor Osvaldo Hurtado. La Declaración y Programa de Acción aprobados en esa Conferencia hacen hincapié en la necesidad de encontrar soluciones potiticas colectivas al problema del endeudamiento, que repartan de manera más proporcionada los costos del refinanciamiento. También subraya la Declaración de Quito la necesidad de ir más allá y fomentar la cooperación entre paises latinoamericanos en la búsqueda de cambios estructurales de más largo plazo en los campos del comercio, la industria, la agricultura, etc. Se reactiva así un amplio debate sobre los temas fundamentales que determinan el futuro de la región y su inserción externa, y con ello la cripsl. vuelve a tomar el papel que fue en otros tiempos el suyo, de estímulo intelectual y como lugar de intercambio de ideas. 


\section{II \\ Las perspectivas de salida de la crisis en el Norte y a nivel internacional}

Los documentos surgidos a partir de aquella Conferencia subrayan en forma adecuada que las estrategias que se elaboren para el desarrollo latinoamericano tienen que referirse a las perspectivas del sistema mundial que, de muchas maneras, van a tener impacto en la situación de la región. Por consiguiente parece indispensable ensayar algún ejercicio de prospectiva internacional, a lo menos en forma conceptual, con miras a discutir las posibilidades de ampliar esa reflexión y darle ulteriormente un marco cuantitativo.

A estas alturas me limitaré a presentar en forma crítica dos hipótesis acerca de la solución de la crisis que, me parece, sintetizan de manera esquemática las ideas que circulan comúnmente en los países del Norte.

Las hipótesis siguientes no son previsiones más o menos probables de la evolución de los paises del Norte hasta el año 2000. Representan sólo un esfuerzo por exponer coherentemente posibles variantes de esa evolución, con el objeto de proporcionar a las autoridades responsables de los países del Sur un marco de referencia para el estudio de sus propias estrategias de desarrollo.

Antes de presentar las hipótesis, es preciso subrayar que no existe ninguna garantía que el sistema internacional encuentre soluciones en una u otra de ellas. Es posible que no se superen de manera durable las contradicciones actuales, y que persistan fenómenos como la cesantía, los desequilibrios entre sectores y regiones y, quizá, una nueva recesión. La situación de Europa tiende a mostrar que tal evolución es posible, y no hay que descartar un nuevo retroceso en Estados Unidos. En tales casos, podrían permanecer por largo tiempo más, fenómenos de fraccionamiento entre paises del Norte, tendencias al proteccionismo, y tensiones internacionales agravadas por el alto nivel de los gastos en armamento.

En tales circunstancias, todos los paises, y más aún los paises en desarrollo, sufririan una recesión prolongada y tendrían que elaborar es- trategias de sobrevivencia adaptadas. Sin desestimar esas eventualidades me parece más interesante estudiar el caso de salidas de crisis efectivamente realizadas con cierto grado de éxito. Vamos a hacerlo a continuación en dos hipótesis presentadas de manera esquemática.

\section{Primera hipótesis: salida de la crisis por la vía tradicional del capitalismo liberal}

En esta hipótesis se parte del supuesto básico que la política económica de la administración Reagan ha logrado ya la salida de Estados Unidos de la crisis. La incorporación de las tecnologias más modernas en las principales industrias, hace posible nuevos progresos en la productividad después de un largo período de estancamiento. La flexibilización de las regulaciones en varios sectores y el desmantelamiento de los sistemas rígidos del "Estado del Bienestar" dan un nuevo dinamismo a las empresas. Se registran, así, un crecimiento sin inflación del producto bruto y una reducción significativa del subempleo.

En este supuesto, la aplicación continuada de esas políticas resulta a la postre en un desmantelamiento más profundo del "Estado del Bienestar". La esfera del mercado se extiende a actividades de tipo social que estaban antes a cargo del Estado (salud, educación, comunicaciones, correo, posiblemente aun servicios de seguridad). De ese modo se amplía el campo de la acumulación capitalista y se integran sectores de alta productividad con sectores de consumo de masas. Surgen nuevos patrones de consumo, que se difunden poco a poco, $y$ proporcionan reservas de crecimiento por un período largo. A ese modelo de crecimiento acompañan grandes desigualdades importantes entre regiones y grupos sociales.

En esa hipótesis las empresas transnacionales norteamericanas, sin el contrapeso del Estado o la sociedad civil, fortalecen su poder, extienden su espacio de acción y aumentan el nivel y eficiencia de sus operaciones. El Estado norteamericano 
favorece esas actividades en el mercado interior y las apoya en los países extranjeros.

La realización estable y permanente de ese escenario en Estados Unidos presupone la solución de los problemas del déficit del presupuesto federal de $\mathbf{2 0 0}$ mil millones de dólares y del comercio exterior de 100 mil millones de dólares que encara actualmente el país, lo que demuestra que no se puede considerar como fácilmente alcanzable.

A nivel internacional, la realización de esa hipótesis supone que el modelo de crecimiento y el patrón de consumo elaborados en Estados Unidos se extienden a los demás países industrializados. Es probable que Europa tendría muchas dificultades en adaptarse, ya que eso significaría de hecho el desmantelamiento de los sistemas tradicionales de protección social. Tal evolución provocaría tensiones y conflictos, que tendrían impacto en la competitividad de las industrias europeas, y en la influencia y presencia de ese continente frente a Estados Unidos y Japón tan to a nivel mundial como en el mismo mercado europeo.

En esa hipótesis, Japón sería un partenaire privilegiado del polo dominante norteamericano, aunque la introducción del nuevo modelo tropezaría con dificultades de tipo cultural. Algunos países del Sudeste asiático podrían participar de manera activa en el modelo norteamericano-japones y el peso principal de la economía mundial se trasladaría más y más a la zona del Pacífico.

En dicha situación el sistema internacional funciona sin mayores cambios institucionales. Los organismos multinacionales tienen un papel de escasa importancia. El sistema monetario se organiza basado en la dependencia de un dólar fuerte. Se trata, en resumen, de un mundo que se integra bajo el dominio de las transnacionales norteamericanas apoyadas por el poder político de Estados Unidos.

En la sección siguiente examinaremos las posibilidades que se les presentan a los paises en desarrollo de elaborar estrategias de desarrollo a b sse de las hipótesis estudiadas. Es evidente que elı este primer supuesto tienen un margen de au ción muy reducido para resistir al dominio economico y político. El patrón de consumo dominante continuaria extendiéndose a los grupos so iales que perciben ingresos adecuados. El sis- tema productivo del Sur participaría en el sistema internacional en los sectores y países que las transnacionales consideraran convenientes para la maximización de sus ingresos a nivel mundial. Se trataría fundamentalmente de los sectores tradicionales de la minería y la agricultura y de algunos sectores industriàles básicos o de uso intensivo de mano de obra que podrían incorporarse a la división internacional del trabajo. Parece inevitable en tal perspectiva que se profundicen las desigualdades y dislocaciones entre los países del Tercer Mundo y, dentro de cada país, entre los sectores integrados al mercado mundial y los sectores marginados de él.

\section{Segunda hipótesis: salida de la crisis por la vía socialdemócrata}

Se supone aquí que la política esbozada en el escenario anterior, después de unos años llega a una impasse aun en Estados Unidos y que se buscan sistemas más equilibrados entre un capitalismo flexible, en que las transnacionales siguen siendo fuertes, y Estados de tipo socialdemócrata. Se trataría de formas nuevas, distintas del clásico "Estado del Bienestar", capaces de organizar la demanda social mediante servicios públicos eficientes, de realizar cierta redistribución del ingreso entre regiones y grupos sociales y de encontrar soluciones innovadoras al problema del subempleo. El patrón de consumo sería bastante distinto del planteado en la hipótesis anterior; habría mayor desarrollo de los servicios colectivos, organización diferente del tiempo entre el trabajo, la vida asociativa y el tiempo libre. Se puede de hecho encarar varios patrones distintos, los unos con uso relativamente importante de bienes y servicios tradicionales, los demás más innovadores en que el desarrollo de la sociedad civil fuera del mercado llegara a modelos de crecimiento cercano al "crecimiento cero".

A nivel internacional, es posible imaginar distintas variantes según la extensión geográfica de ese tipo de solución. Una se trataría de la extensión, en formas diversas, a todos los países del Norte, con una gestión compartida y equilibrada en el sistema internacional. Otra, representaría una situación más conflictiva en que Europa se adaptaría más fácilmente a sistemas cercanos a su organización social tradicional y Estados Unidos tardaría en encontrar una solución 
adecuada y permanecería por más tiempo en la crisis.

De todos modos, esos modelos implican la organización de la economía de mercado con poderes de contrapeso (el Estado y la sociediad civil). A nivel internacional significarían instituciones internacionales fuertes capaces de controlar el sistema en los campos monetario, financiero y comercial. Además, crearían las condiciones necesarias para la aplicación de medidas similares a las propuestas en el informe Brandt y varias resoluciones de las Naciones Unidas: organización de los mercados de materias primas, sistemas de preferencia, aumento de las transferencias de tecnologia, ayuda intensa y generalizada, y reestructuración industrial a nivel mundial mediante una concertación internacional.

La pregunta que cabe hacerse aquí es si, aun en la hipótesis aparentemente muy optimista de una aplicación amplia de ese tipo de sistema, ésta significaría un verdadero desarrollo de los paises del Sur. Mi opinión personal es que, si no se acompaña de la adopción de medidas determinadas por parte de esos mismos paises, en favor de un desarrollo autónomo, ese tipo de sistema internacional, aunque más favorable que el sistema antes descrito, no alcanzaría de hecho los objetivos que se proponen sus partidarios. Existen grandes riesgos que una estrategia internacional de esa naturaleza conduzca a una fuerte dependencia, y a desigualdades mayores entre paises y entre grupos sociales. La incorporación selectiva al mercado internacional podría significar en una parte mayoritaria del Tercer Mundo dislocaciones internas, marginación y proletarizacion crecientes.

Considero conveniente a ese respecto reflexionar sobre las soluciones a la crisis basadas en esquemas de aumento masivo de los flujos financieros Norte-Sur, calificadas a veces de nuevo Plan Marshall hacia el Tercer Mundo. Tales esquemas subrayan la interdependencia NorteSur y pretenden resolver conjuntamente los problemas de la cesantía en el Norte y la falta de inversiones en el Sur. Antes de embarcarse en tales planes sería preciso analizar to que pasó en los últimos años del decenio de 1970 y los primeros del de 1980. La abundancia de liquidez en el Norte (en parte petrodólares), combinada con la recesión, resultó en un aumento de los flujos financieros Norte-Sur a niveles muchas veces más altos que en los propuestos planes Marshall. De hecho, produjo un fuerte incremento de los flujos comerciales (bienes suntuarios, armamento, y algunos bienes de capital), y fue muy útil para las industrias del Norte amenazadas por la recesión. Pero la abundancia de recursos significó para el Sur la competencia de los bienes im. portados que debieron encarar las industrias nacionales; proyectos faraónicos; desarticulaciones economicas y sociales y endeudamiento gigantesco. La interdependencia obró claramente de ma* nera unilateral.

La historia de los últimos años nos invita a reflexionar sobre lo inadecuado de las soluciones basadas en transferencias financieras. Las dificultades con que tropezaron en su desarrollo los países que disponen de recursos provenientes de renta (renta petrolera o de otras fuentes) of recen un tema de reflexión en el mismo sentido.

Cabe esperar que el análisis de la situación presente contribuya a que se tenga una conciencia más clara de que el financiamiento internacional, que ha sido durante largos años el punto central de todo el debate sobre el desarrollo, es de hecho un arma de doble filo. Es digna de mención a ese respecto la noticia que el Banco Mundial va a tener que reducir en $1985 \mathrm{el}$ nivel de sus préstamos debido a la falta de demanda por parte de los países en desarrollo. Sería interesante ver si esa tendencia se mantiene en el futuro. 


\section{¿En la búsqueda de un nuevo paradigma?}

De las reflexiones anteriores se concluye que los países en desarrollo necesitan un nuevo patrón de desarrollo o, según una formula que se ha usado en América Latina en los últimos años, nuevos estilos de desarrollo. No quiero entrar ahora en una controversia sobre el punto tocado por Enrique Iglesias en su presentación a la reunión de la CEPAL en Lima en 1984: si es prematuro o no buscar un paradigma económico para América Latina (Iglesias, 1984). El análisis de la crisis y los efectos posibles de las distintas hipótesis examinadas antes, inducen más bien a prestar nuevamente atención a ideas que se desarrollaron en los años setenta y que a mi modo de ver no han perdido vigencia. Las politicas neoliberales aplicadas según la 'nueva ortodoxia' han demostrado sus efectos perversos. Han empeorado en forma desmesurada los defectos estructurales de dependencia, desigualdad, dislocación económica y social, ataque al medio ambiente, característicos del subdesarrollo.

Aunque las fuertes restricciones vinculadas al endeudamiento creen condiciones más difíciles, parece más evidente que nuncá la necesidad de destigar a los países en desarrollo de los efectos negativos del mercado internacional. La crisis pone en la orden del día la búsqueda de estrategias de desarrollo autónomo, autosostenido. Esos conceptos acuñados en los años sesenta por investigadores del Tercer Mundo, se incorporaron a la reflexión y al debate internacionales a mediados del decenio de 1970 . Tuvieron expresión pública en documentos de la Fundación Hammarskjöld o en la reunión de Cocoyoc. También pasaron por textos oficiales del Sistema de las Naciones Unidas como, por ejemplo, de la Sexta Sesión Extraordinaria de la Asamblea General (mayo 1974), la Conferencia de Lima sobre la industrialización (marzo 1975), la Conferencia de la ort sobre las necesidades básicas (mayo 1976).

Los acontecimientos de los diez años siguientes no han quitado validez (muy por el contrario) a las conclusiones aprobadas en esa época como, para mencionar algunas de ellas: el desarrollo es distinto del puro crecimiento económico, pero implica cambios estructurales. Esos cambios no se realizarán solamente por el juego de las fuerzas del mercado; necesitan acciones deliberadas de redistribución de la producción, de la riqueza, del poder, tanto al nivel nacional como al nivel internacional.

En el campo industrial, la comunidad internacional adoptó un objetivo cuantitativo de reestructuración del sector a nivel mundial y decidió establecer un sistema permanente de consultas para tomar medidas adecuadas a dicha reestructuración.

El desarrollo implica satisfacción prioritaria de las necesidades básicas y para eso políticas apropiadas de redistribución del ingreso.

El problema esencial de esas estrategias de desarrollo no es que fueran erróneas, sino que no se aplicaron en la práctica. Aun en países donde se proclamaron esos principios como base teórica de las políticas nacionales, se pueden comprobar muchas contradicciones entre esos principios y las medidas efectivamente tomadas.

Hoy, igualmente, los países del Tercer Mundo tratan de conquistar un grado de autonomía que les permita decidir, según sus propios objetivos de desarrollo, acerca de sus patrones de consumo, sus producciones, la elección de tecnologlas, sus inversiones, y el uso de sus capacidades financieras. Eso es posible sólo si disponen al mismo tiempo de cierto grado de autonomía en su inserción en el sistema internacional, y en la definición de sus políticas comerciales, financieras y monetarias.

En las condiciones actuales esas estrategias pueden parecer utópicas. La mayoría de los países encontrarían dificultades insuperables al aplicarlas en forma aislada. Pero podrían ser más factibles si se organizara la cooperación Sur-Sur en los campos agrícola e industrial y si se establecieran instituciones propias de regulación comercial, financiera y posiblemente monetaria. Es evidente que el contexto internacional puede ser favorable en mayor o menor grado a la realización de estrategias de autosustentación. Así, por ejemplo, entre las salidas de crisis mencionadas en la sección anterior, las soluciones del segundo 
tipo harian menos difícil el desarrollo autónomo que las soluciones del primer tipo.

De todos modos, el problema presente no consiste, a mi modo de ver, en definir un nuevo paradigma sino en dar al concepto de desarrollo autosustentado un contenido operacional que pueda materializarse en decisiones precisas o medidas bien definidas de política económica. El contenido de esos objetivos y su articulación entre sí tienen obviamente que ser distintos de un país a otro. En general, habría que buscar en cada caso los puntos claves más importantes, los núcleos decisionales en que se concentren el esfuerzo, las medidas, las acciones. A título de ejemplo, se mencionan algunos puntos claves que parecen de importancia en la mayoría de los países latinoamericanos.

-El logro de la autosuficiencia en alimentos. Este punto se relaciona con los objetivos de superación de la dependencia, modificación de los patrones de consumo, satisfacción de las necesidades esenciales, etc. Atañe al sector rural, que tendría que ser reestructurado en profundidad. También afecta las relaciones exteriores posición frente al agrobusiness mundial, cooperacion internacional), la estrategia de industrialización, las relaciones campo-ciudad, etc. Dar a este objetivo máxima prioridad, no en forma retórica, sino fijando un proceso concreto, con niveles y fechas, puede significar una reorientación cabal de la política económica y social en sui conjunto,

-la definición del proceso de industrialización a partir de un núcleo endógeno (Fajnzylber, 1983) mediante una dinámica progresiva de autonomía creciente. Eso implica no sólo metas de producción y programas de inversiones, sino politica y sistema institucional de control de la importación de tecnologías (cf., la política mexicana de los años setenta y la decisión 24 del Pacto Andino), instituciones de investigación y de ensenanza técnica, proyectos de producción de bienes de capital, etc.; eso significa también la integración horizontal de todas las actividades sectoriales.

- la articulación en los sectores agrícola e industrial de las empresas de tecnología moderna con las empresas tradicionales. El deterioro de esa articulación es una causa mayor del subdesarrollo, de la desigualdad social y tambien regional y de la transformación de las empresas modernas en 'enclaves' del sistema internacional. El progreso en esa materia supone no sólo la dictación de medidas internas para cada sector sino el control estricto de la relación con el exterior, la orientación hacia el mercado interno, el control de los precios, la adopción de medidas de incentivos fiscales, programas de formación, etc.

Al tomar esos ejemplos no fue mi intención entrar a analizar cada uno de los temas sino indicar que no son de índole sectorial, que deba dárseles un tratamiento fraccionado. Cada uno combina elementos externos e internos, aspectos horizontales y verticales, y medidas sectoriales, giobales e institucionales.

La preocupación de dar un contenido concreto a objetivos que quedaron muchas veces en el pasado en el plano ideologico o aun retórico, lleva a reflexionar sobre nuevas formas de planificación y de cooperación.

\section{Nuevas formas de planificación}

La planificación, que tuvo un perfodo de auge en los años sesenta tanto en Europa como en América Latina, ha atravesado después un periodo largo de decadencia. Parece paradójico querer reimplantarla en momentos mucho más difíciles, en que la incertidumbre del futuro es máxima y los gobiernos están sometidos a la presión continua de tener que tomar decisiones urgentes a muy corto plazo. Pienso, sin embargo, que si los tiempos de crisis hacen las cosas más difíciles, las hacen también más necesarias. Debido a que requieren solucionar los problemas inmediatos y a la vez elaborar estrategias nuevas, se ven obligados a concebir sistemas de planificación renovados en sus instituciones, procesos y métodos.

Presentaré, a ese respecto, algunas sugeren- 
cias, a partir de un examen crítico de los métodos elaborados en los periodos anteriores. Es cierto que esos métodos no captaron cabalmente las conclusiones lógicas de los principios proclamados acerca de la naturaleza del subdesarrollo y las estrategias necesarias para superarlo. Muchos planes no dieron un contenido concreto a la declaración del principio que el desarrollo no es sólo crecimiento económico; y de hecho limitaron sus objetivos a la consecución del crecimiento más rápido posible.

Los instrumentos técnicos utilizados adolecen en general de las mismas limitaciones: los modelos de optimación global son a nivel nacional o sectorial; los análisis de costo-beneficio de los proyectos se proponen la maximización del producto, es decir, un objetivo aritmético y no estructural.

Para superar la dicotomía existente no basta incorporar en los objetivos cifras de producto interno bruto, índices de producción o niveles de inversión. Es preciso dar un contenido a conceptos como el de 'estilos de desarrollo' en que la reflexión teórica ha progresado en América Latina en los últimos años. Es decir, que los distintos aspectos de una estrategia: patrones de consumo, estructura de los ingresos, articulación de los sectores, elección de las tecnologías, relación con el exterior, identidad cultural, se interrelacionan. Por lo tanto, el sistema de planificación, el proceso de construcción de los planes, los instrumentos técnicos utilizados, y las instituciones de planificación, deben organizarse en consecuencia.

Por consiguiente se trata de concebir y construir un sistema de planificación que ayude efectivamente al proceso de toma de decisiones en las materias complejas que mencionamos antes, incorporando objetivos múltiples no ponderables entre sí; y, además, que sea flexible, capaz de adaptarse rápidamente a los cambios bruscos que afectan las condiciones externas e internas. Me limitaré a algunas reflexiones sobre las características de tales sistemas.

\section{Articulación de los horizontes temporales}

Los sistemas de planificación privilegiaron durante mucho tiempo la construcción de planes de mediano plazo. Hoy es necesaria una planificación que sea a la vez más estratégica y más opera- cional, es decir, que logre una integración más estrecha entre los objetivos estructurales, que tienen usualmente efecto a largo plazo (15 6 20 años) y las decisiones con las inherentes medidas de política económica, que se toman hoy. A ese fin considero importante extender los instrumentos de planificación en ambas direcciones: hacia el largo plazo y hacia el corto plazo.

A largo plazo se trata de elaborar instrumentos prospectivos capaces de proyectar hasta fines de siglo la situación del sistema mundial y las consecuencias de las estrategias nacionales y regionales. Se incluyen aquí proyecciones cuantitativas, única manera de asegurar un grado mínimo de coherencia en la evolución de varias magnitudes. Eso no significa que ese esquema de largo plazo tenga las mismas clasificaciones y los mismos detalles que el de mediano plazo.

Esos instrumentos prospectivos permitirían estudiar las consecuencias de estrategias sustitutivas en varias hipótesis sobre el porvenir del sistema internacional. Sería de gran ayuda para estudiar la efectividad del ejercicio y las posibilidades de cooperación entre los países latinoamerícanos que los distintos paises se pusieran de acuerdo para definir hipotesis comunes sobre el contexto internacional. Asi, se podría comparar estrategias y cotejar sus compatibilidades. Este podría ser un primer paso, bilateralmente o a nivel regional, hacia la armonización de las estrategias.

Paralelamente a la extensión hacia el largo plazo es muy importante en las condiciones presentes elaborar instrumentos para la ejecución permanente de los planes, que se adapten rápidamente a los cambios que se producen en la situación internacional o interna. La experiencia de los planes anuales operativos (a veces llama* dos también presupuestos económicos) es muy valiosa en ese sentido, aunque en la situación actual surjan otras dificultades. Ocurren cambios muy bruscos en las tasas de inflación, la balanza de pagos, el nivel de cesantía, y los tipos de interés; es muy difícil, entonces, evaluar los comportamientos del consumo, el ahorro, la inversión, y las decisiones de producción, resultantes de esos cambios.

De lo anterior se deducen varias consecuencias prácticas.

- La necesidad de un sistema mucho más ágil 
de recopilación, circulación y análisis de la información económica que se requiere para el proceso de toma de decisiones.

- La elaboración de instrumentos de previsión adaptados a situaciones muy diferentes de las del pasado, quita mucha validez a los modelos basados en las relaciones de comportamiento históricas.

- La importancia de una vinculación más estrecha entre las instituciones que participan en las decisiones de política económica y, por consiguiente, en la elaboración de los planes anuales que ayudan a tomar esas decisiones (en particular organismos de planificación, hacienda y bancos centrales).

\section{Articulación de la planificacion global con la planifícación sectorial}

Los métodos tradicionales de planificación se caracterizan muchas veces por la débil vinculación que existe entre la planificación global y la planificación de los varios sectores a nivel tanto conceptual como institucional. La primera proporciona un esquema bastante impreciso de la estructura general de la demanda. De hecho, la planificación de cada sector se hace en forma casi autónoma en los ministerios correspondientes; en los sectores de gran concentración, las grandes empresas tienen un papel importante y el plan nacional es a menudo la presentación conjunta, más o menos coordinada, de las perspectivas de los productores.

Un ejemplo ilustrativo es el sector de la energía, en que la planificación se limita a veces a los subsectores petrolero y eléctrico. En muchos paises, por recomendación del Banco Mundial, se hacen ejercicios de planificación energetica fuera del contexto de la planificación global.

Ineludiblemente, esos planes sectoriales, e incluso subsectoriales, se preocupan sólo de maximizar el producto parcial, siguiendo criterios de optimación propios de un solo sector o a veces de la empresa responsable de un solo subsector. De ese modo se profundiza la desarticulación de las economías latinoamericanas y se multiplican los efectos externos negativos. Como lo vimos antes, los objetivos de un verdadero desarrollo no atañen a un solo sector sino que se refieren a varios sectores y a las relaciones entre ellos, postulado que los métodos de planificación sectorial y su organización institucional deben tener en cuenta. La organización de los estudios y el proceso de toma de decisiones deben permitir optar entre soluciones sustitutivas que sin dejar de ser optimistas del punto de vista de un sector tengan efectos indirectos positivos en los objetivos generales y el desarrollo de otros sectores.

Para continuar con el ejemplo de la planificación energética, los métodos de planificación tendrían que evitar la repetición de los errores cometidos antes en muchos paises, es decir, la construcción de sistemas energéticos inadecuados tanto a las necesidades de los usuarios como a los recursos de los países. Eso supondría incorporar en la planificación decisiones sobre los usos y el manejo de la energía y no sólo sobre la producción y las inversiones del sector. Significaría, además, reequilibrar el predominio presente del petróleo en países que no disponen de recursos propios, en favor del desarrollo de otras fuentes energéticas nacionales.

Unicamente los métodos que van más allá de los modelos de optimación o del análisis costobeneficio pueden ayudar a tomar decisiones racionales en problemas complejos como, por ejemplo, la electrificación como base del desarrollo de una industria nacional de bienes de equipo; el estudio de los efectos positivos y negativos de un programa de uso energético de ciertos cultivos (Proalcohol); la superación de los efectos negativos de la renta petrolera en el sistema socioeconómico de los países productores, etc.

\section{Proceso de concertación}

Se ha reprochado a la planificación en América Latina de ser excesivamente burocrática y de producir planes preparados sin la participación de los grupos sociales interesados y, en consecuencia, de poco efecto práctico en el manejo ulterior de la política económica.

El feliz surgimiento en la mayoria de los países de gobiernos democráticos da la oportunidad de renovar los sistemas de planificación, e incorporar al proceso una organización de consulta de los grupos sociales. Esto es aún más necesario en la dificil situación actual en que los errores de las políticas económicas del pasado, y su consecuencia más visible,el endeudamiento, van a hipotecar por muchos años el futuro desarrollo de los paí- 
ses. Para elaborar sin embargo estrategias de largo plazo autónomas y defenderlas frente a las presiones del exterior, los gobiernos necesitan el apoyo interno más amplio posible. La construcción de perspectivas nacionales de mediano o largo plazo por medio de un proceso de concertación puede ayudar a encontrar un punto de convergencia de las distintas aspiraciones y aliviar contradicciones que parecen insuperables en el corto plazo.

\section{V \\ Nuevas formas de cooperación}

Tal como el de la planificación, el tema de la cooperación entre países en desarrollo, y más precisamente de la cooperación regional latinoamericana, despertó en una época grandes esperanzas seguidas por muchas desilusiones. No es posible en el marco presente analizar los progresos y retrocesos de los varios esquemas regionales y subregionales latinoamericanos. La evolución política en algunos países del continente y también la ola de politicas ultraliberales han tenido sin duda efectos negativos. Pierde mucho de su razón de ser el esfuerzo de cooperación Sur-Sur, si el desarrollo se busca mediante una inserción indiscriminada en el mercado mundial. A veces incluso empresas extranjeras pueden tener más fuerza y flexibilidad que las nacionales para aprovechar las disposiciones favorables de algunos tratados de integración.

Por el contrario, si se toma el camino que conduce a un desarrollo autosustentado, la cooperación regional y subregional recupera toda su importancia. Tanto a nivel regional como a nivel subregional existe abundancia de textos juridi$\cos$, estructuras institucionales, experiencia acumulada. Sería muy oportuno hacer un análisis histórico-crítico de los varios esquemas, y detectar las causas de sus fallas con el fin de proponer innovaciones que les permitan poner en práctica estrategias nuevas. Adelantaría a estas alturas una sola idea: no faltaron los conceptos, las ideas, los textos, los estudios, ni siquiera en unos casos las instituciones y el personal calificado; faltaron las estrategias de desarrollo adecuadas para aprovechar esos elementos. Los instrumentos de cooperación entre paises pierden su eficiencia si no deciden al mismo tiempo definir posiciones comunes hacia el exterior. Tomaré un solo ejemplo: la famosa decisión 24 del Acuerdo de Cartagena. Hay allí todos los elementos de una cooperación industrial eficiente que va más allá de los aspectos comerciales y financieros y apunta hacia políticas industriales y tecnológicas comunes. $\mathrm{Su}$ defecto no está en su concepción sino en el hecho que nunca ha sido puesta en practica. Podría nuevamente conquistar vigencia si los países miembros se pusieran de acuerdo para definir en común su modo de inserción en el mercado internacional y sus perspectivas de desarrollo de largo plazo. A nivel regional latinoamericano quisiera sólo recordar dos documentos que interesan a sectores importantes, el de la industria y el de la energía, que también podrían ser instrumentos útiles en la situación presente.

En lo industrial, mencionaré la Conferencia Latinoamericana sobre Industrialización que reunió a los ministros de industria en Ciudad de México en noviembre de 1974. De acuerdo con lo recomendado por la Sexta Sesión Especial de la Asamblea General de las Naciones Unidas y en la perspectiva de la Conferencia de Lima que tuvo lugar pocos meses después, la Conferencia aprobó un objetivo cuantitativo para la industria latinoamericana en el marco mundial y afirmó, entre otros, el principio de que el juego sin restricción de las fuerzas del mercado no es el medio más adecuado para promover la industrialización y que los acuerdos de cooperación industrial entre gobiernos y entre empresas tenían que elaborarse con el apoyo del Sistema de las Naciones Unidas.

También a nivel regional la Conferencia propuso objetivos de armonización y coordinación de las polfticas industriales, de especialización y complementación, fortalecimiento de los esquemas de integración existentes y búsqueda de nuevas formas. Resolvió la adopción de medidas para la armonización y coordinación de las políticas de inversion (nacional y extranjera), desarrollo tecnológico, cooperación cientifica y tecnológica, y defensa de los precios de las exportaciones. 
Aprobó el principio de empresas multinacionales latinoamericanas, acuerdos de complementación, y un sistema regional de importaciones. Encomendó a las organizaciones regionales, especialmente a la CEPAL, desarrollar actividades de estudio y de promoción para ayudar a la aplicación de las medidas adoptadas.

Después de transcurridos diez años, hay que reconocer que poco se ha hecho para poner en práctica esas ideas excelentes. Los gobiernos no supieron dar un contenido político concreto a los principios que ellos mismos habían proclamado. Ni la CEPAL ni el SEL.A, recién creado en esa época, aprovecharon las circunstancias favorables y los mandatos claramente recibidos para impulsar acciones determinadas en favor de la cooperación industrial.

Las dificultades creadas por la crisis, si se hace un análisis adecuado de sus causas, pueden inducir a los países latinoamericanos a adoptar nuevos estilos de desarrollo industrial, a partir de los núcleos endógenos que existen en varias partes del continente. En tal caso, las acciones de cooperación industrial en las líneas concebidas hace diez años pueden multiplicar las oportunidades y acelerar el proceso.

En el campo de la energía, también existen posibilidades de cooperación que no se han aprovechado plenamente. Una iniciativa muy interesante es el Acuerdo de San José entre paises productores de petróleo (México y Venezuela) y los países importadores de América Central y del Caribe. Vincula el suministro de petróleo al financiamiento de proyectos de desarrollo, en particular en el campo energético. Sin entrar en un análisis pormenorizado de la aplicación del Acuerdo, cabe recordar que a pesar de sus puntos positivos, registró en el último período una tendencia a una marcha más lenta. Cabe notar también que la última renegociación se hizo en condiciones menos favorables para los paises beneficiarios.

El Programa Latinoamericano de Cooperación Energética (PLACE), aprobado en 1981 por 25 paises en el marco de la ol.ADf., es más ambi- cioso. Se propone desarrollar entre los países una visión estratégica común del aprovechamiento energético, impulsar una acción unificada y permanente, promover la cooperación en todos los campos de la capacidad científico-técnica, de la prospección y exploración, y de la capacitación. El PLACE pretende ir más allá que el intercambio de informaciones, la realización de estudios e investigaciones comunes y la cooperación técnica; propone la creación de industrias comunes y en particular de una industria latinoamericana de bienes de capital y equipos para el sector energetico.

Después de tres años de vida, las realizaciones del PLACE han sido bastante decepcionantes. Los proyectos en marcha se quedan en un nivel limitado. Representan en gran parte actividades de estudio, capacitación y asistencia técnica. Los proyectos concretos interesan a países individuales y no tienden a desarrollar capacidades productivas comunes a varios países. Habría que preguntarse si después de aprobar el Programa, los gobiernos mostraron una verdadera voluntad de ponerlo en marcha. Pero también se puede notar que la Secretaría de OLADE dedicó mayor esfuerzo a trabajos técnicos y metodológicos (como balances energéticos, métodos de previsión de la demanda y de la oferta, seminarios de capacitación, etc.), campos en que actúan ya de manera muy eficiente varios institutos de investigación en América Latina. Mientras, dejó de lado la tarea política de promover entre los gobiernos miembros proyectos $y$ empresas comunes. Sin embargo esas perspectivas quedan abiertas, y por muchas razones el sector energético es uno de los que más se presta a un avance rápido de la cooperación latinoamericana.

Estas líneas expresan la convicción que pese a la prepotencia manifestada por la ideología dominante, la nueva ortodoxia, el momento es muy propicio para reflexionar en profundidad sobre una nueva etapa del desarrollo latinoamericano. La presencia de gobiernos democráticos en la mayoría de los paises, la búsqueda de un nuevo rumbo manifestada en particular a partir de la reunión de Quito muestran esas posibilidades.

\section{Bibliografia}

Fajnzylber, Fernando (1983): La industriatización trunca de América Latina. México: Editotial Nueva Imagen.
Iglesias, Enrique (1984): América Latina: Crisis y opciones de desarrollo. Revista de la cEpal, $\mathbf{N}^{\mathrm{O}} 23$. Agosto. 